\title{
Management of acute pyelonephritis in the emergency department observation unit
}

Kathleen Swee Min $\underline{K h o o}^{1}$, MBBS, MrCEM, Zhen Yu $\underline{\operatorname{Lim}}^{2}$, MBBS, Chew Yian $\underline{C h a i}^{3,4}$, MBBS, MRCEM, Malcolm Mahadevan ${ }^{3,4}$, MBBS, FRCS, Win Sen $\underline{K u a n}^{3,4}$, MRCS, MCI

INTRODUCTION This study aimed to assess the effectiveness of the emergency department observation unit (EDOU) for patients with acute pyelonephritis in a Singapore tertiary academic medical centre.

METHODS We reviewed the clinical records of consecutive patients who presented with pyelonephritis between 1 July 2012 and 31 October 2014 to collect information on demographics, symptoms, signs, laboratory and radiological results, treatment, and clinical outcomes.

RESULTS Of 459 emergency department (ED) patients who were identified as having pyelonephritis, 164 (35.7\%) were managed in the EDOU. Successful management in the EDOU was achieved in $100(61.0 \%)$ patients. Escherichia coli was the predominant $(64.6 \%)$ micro-organism in urine cultures and was positive in 106 patients. Patients diagnosed with acute pyelonephritis who were successfully managed in the EDOU had a lower incidence of nausea $(32.0 \%$ vs. $60.9 \%$, $p<0.001)$ and vomiting $(15.0 \%$ vs. $50.0 \%, p<0.001)$ compared to those who were not successful.

CONCLUSION EDOU is useful for both observation and treatment of patients with acute pyelonephritis. Urine cultures are sufficient for the identification of the culprit micro-organism. Patients who present with prominent symptoms of vomiting should have routine administration of antiemetics, while consideration for second-line antiemetics is recommended for those with persistent symptoms.

Keywords: emergency service, hospital, observation unit, pyelonephritis

\section{INTRODUCTION}

Acute pyelonephritis, an illness caused by bacterial infection of the kidney, is suggested by the presence of flank pain, nausea or vomiting, fever, and costovertebral angle tenderness with or without the typical symptoms of cystitis, and is confirmed by the finding of bacteriuria in the setting of these symptoms. ${ }^{(1)}$ It occurs in more than 250,000 adults in the United States yearly, costing an estimated USD 2 billion a year. ${ }^{(2,3)}$

The recommendations for treatment vary from inpatient management to outpatient therapy for different groups of patients. ${ }^{(4-8)}$ Older studies have shown that observational care is feasible in managing acute pyelonephritis and reducing hospital admissions. ${ }^{(9,10)}$ Given the known complications of septic shock, multi-organ failure and death in severe cases, patient care can be optimised by appropriate unit selection, which can reduce morbidity and mortality and allow limited hospital resources to be used more judiciously.

The primary aim of this study was to assess the effectiveness of the emergency department observation unit (EDOU) for patients with acute pyelonephritis, defined as successful discharge from the EDOU without re-admission within seven days. The secondary aim was to identify the risk factors and predictors for requiring subsequent inpatient management in patients with pyelonephritis admitted to the EDOU, in order to improve the effectiveness of the EDOU.

\section{METHODS}

A retrospective chart review was conducted of consecutive adult patients presenting to the emergency department (ED) of National University Hospital, Singapore, with a primary or secondary diagnosis of pyelonephritis between 1 July 2012 and 31 October 2014. Ethical approval was obtained from the National Healthcare Group Domain Specific Review Board (2014/00791), which granted waiver of informed consent.

The EDOU at our institution is a 16-bed facility that is under the oversight of an ED physician for 24 hours daily. A variety of EDOU protocols are available on the department intranet, one of which is the acute pyelonephritis protocol (Appendix). The protocol was created in tandem with infectious disease physicians based on expert opinion. Patients with suspected acute pyelonephritis can be admitted to the EDOU and managed for up to 24 hours. Within 24 hours, patients may be discharged home to continue outpatient treatment if their vital signs are stable and they are able to take well orally. Patients are admitted to an inpatient unit if they turn unwell with unstable vital signs, deteriorating renal function, increasing lactate, white blood cell count $>20 \times$ $10^{9} / \mathrm{L}$, persistent vomiting or inadequate oral intake, increasing loin pain, pyuria, or ballotable kidneys.

Cases were identified using EDOU and inpatient unit primary and secondary discharge diagnosis codes according to the International Classification of Diseases, Tenth Revision, Clinical

\footnotetext{
${ }^{1}$ Acute and Emergency Care Centre, Khoo Teck Puat Hospital, ${ }^{2}$ Department of Medicine, ${ }^{3}$ Emergency Medicine Department, National University Hospital, National University Health System, ${ }^{4}$ Department of Surgery, Yong Loo Lin School of Medicine, National University of Singapore, Singapore

Correspondence: Dr Kathleen Swee Min Khoo, Associate Consultant, Acute and Emergency Care Department, Khoo Teck Puat Hospital, 90 Yishun Central, Singapore 768828. khoo.kathleen.sm@ktph.com.sg
} 
Modification (ICD-10-CM - N10, N11*, N12, N13* and A41.9). A total of $705 \mathrm{ED}$ visits were identified, and all visit records were located and reviewed.

Identified patients were screened for enrolment by a single reviewer (Khoo KSM) who reviewed electronic and physical case records, with $20 \%$ of cases verified independently by a second reviewer (Kuan WS) for accuracy. The second reviewer provided independent input for borderline cases with ambiguous documentation in the medical records or inconclusive comments in reports of imaging modalities. The reviewers were not blinded to the purpose of the study. Patients with at least two out of the following three inclusion criteria ${ }^{(11)}$ were recruited into the study: (a) flank pain or costovertebral angle tenderness; (b) urinary symptoms or bacteriuria/pyuria; and (c) fever. Subjects were also included if they were labelled as having pyelonephritis based on imaging (ultrasonography of the kidneys or computed tomography of the kidneys, ureters and bladder) or by the managing team. Patients who were below 18 years of age, had transplanted kidneys or cancelled their visits before being seen by a physician were excluded. Each ED visit was treated as a separate episode. A total of 459 ED visits satisfied the aforementioned criteria (Fig. 1).

All data was recorded using a standardised abstraction form on Microsoft Excel 2013 (Microsoft Corp, Redmond, WA, USA). Data was collected using an electronic medical record system. Where electronic charts were unavailable, paper charts were retrieved and reviewed.

Data evaluated included demographic information, presenting symptoms, physical findings, laboratory results, imaging, therapy and disposition.

EDOU success was defined as the discharge of a patient who was treated in the EDOU without any repeat hospital admission in the following week. EDOU failure referred to cases in which patients required hospitalisation despite treatment in the EDOU, or returned and were hospitalised within one week.

Upon completion of electronic data collection, charts were reviewed for missing or duplicate data and verified. Data was exported to Stata 14 (StataCorp LP, College Station, TX, USA) for statistical analysis. Categorical variables were reported as frequency and percentage values. For continuous variables, mean \pm standard deviation and median (interquartile range [IQR]) were reported for normal and skewed distributions, respectively. Differences in categorical variables between the EDOU success and failure groups were compared using chi-square test or Fisher's exact test. Normally distributed continuous outcomes were compared using Student's t-test, whereas highly-skewed continuous outcomes were compared using Mann-Whitney $U$ test. Multivariate logistic regression analysis was performed to determine the characteristic differences between the two groups using odds ratios (ORs) and 95\% confidence intervals (Cls). A stepwise approach was used while including only variables with $\mathrm{p}<0.10$ into the model.

\section{RESULTS}

The majority of the 459 patients were female (88.9\%) and young, with a median age of 36 (IQR 28-50) years (Table I). Most

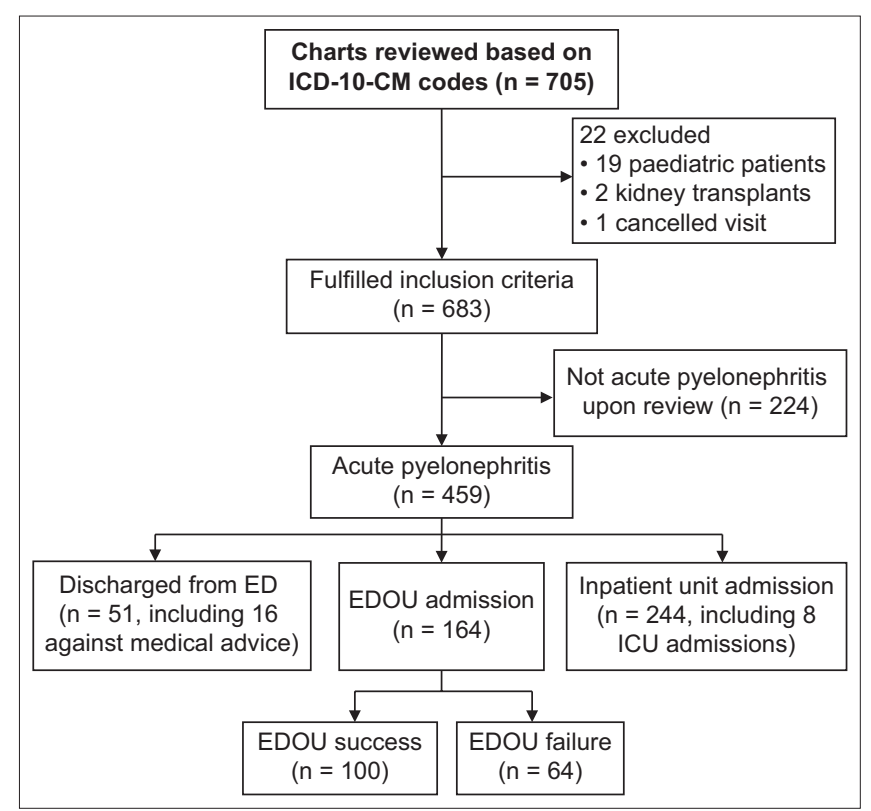

Fig. 1 Flowchart shows the enrolment process. ED: emergency department; EDOU: emergency department observation unit; ICD-10-CM: International Classification of Diseases, 10th Revision, Clinical Modification; ICU: intensive care unit

(81.7\%) had no previous episodes of pyelonephritis. Patients reported having symptoms for a median of 3 (IQR 2-5) days before attending the ED. Slightly more than half (53.7\%) had a urine culture that was positive for Escherichia coliand a minority (3.0\%) were positive for Klebsiella pneumoniae (Table II).

210 patients were admitted to the EDOU under the acute pyelonephritis protocol. 46 (21.9\%) of these patients were found to have other diagnoses such as lower urinary tract infection $(n=25)$, renal colic $(n=5)$, musculoskeletal pain $(n=5)$, gastroenteritis $(\mathrm{n}=3)$, non-specific abdominal pain $(\mathrm{n}=3)$, appendicitis $(n=2)$, dengue $(n=2)$ and gastritis $(n=1) .9(19.6 \%)$ of these 46 patients were admitted to the general ward, while the rest were discharged from the EDOU.

Of the 164 patients admitted to the EDOU with an adjudicated diagnosis of acute pyelonephritis, 100 (61.0\%) were discharged, while $61(37.2 \%)$ were admitted to the inpatient wards, of whom $3(1.8 \%)$ required high dependency care (Table III). Another 3 (1.8\%) patients were discharged against medical advice. The main reasons cited in the clinical records for admission to the inpatient wards were unresolved fever $(53.1 \%)$, pain $(25.0 \%)$, inadequate oral intake or vomiting $(20.3 \%)$, haemodynamic instability $(12.5 \%)$, uptrending total white cell count $(3.1 \%)$, bacteraemia $(3.1 \%)$ and social reasons (1.6\%). Some patients had more than one reason cited. Among the 13 patients who were admitted to the general ward due to inadequate oral intake or vomiting, 12 had metoclopramide administered and two had ondansetron added on in the EDOU as a second-line antiemetic.

Lower systolic blood pressure at triage, fever, higher urea levels and a higher white blood cell count all had a statistically significant association with EDOU failure (Table III), but they were not clinically significant. A lower proportion of patients in the EDOU success group had nausea $(32.0 \%$ vs. $60.9 \%$, p < 0.001$)$ and vomiting $(15.0 \%$ vs. $50.0 \%$, p < 0.001). Multivariate 
Table I. Baseline demographics, symptoms, signs and disposition of the patients ( $n=459)$.

\begin{tabular}{|c|c|c|c|}
\hline Variable & No. (\%) & Variable & No. (\%) \\
\hline Age* $^{*}(y r)$ & $36(28-50)$ & Fever & $433(94.3)$ \\
\hline Female & $408(88.9)$ & Chills & $158(34.4)$ \\
\hline Ethnicity & & Nausea & $203(44.2)$ \\
\hline Chinese & $216(47.1)$ & Vomiting & $148(32.2)$ \\
\hline Malay & $112(24.4)$ & Dysuria & 347 (75.6) \\
\hline Indian & $49(10.7)$ & Abdominal pain & $220(47.9)$ \\
\hline Eurasian & $1(0.2)$ & 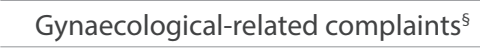 & $17(3.7)$ \\
\hline Others & $81(17.6)$ & Duration of symptoms* (day) & $3(2-5)$ \\
\hline History of pyelonephritis & $85(18.5)$ & Site of flank pain & \\
\hline Previous UTI & $141(30.7)$ & Right & $248(54.0)$ \\
\hline Recurrent UTI ${ }^{\dagger}$ & $62(13.5)$ & Left & $171(37.3)$ \\
\hline Previous admission for pyelonephritis & $81(17.6)$ & Both & $39(8.5)$ \\
\hline Episodes of recurrent pyelonephritis & & Underwent outpatient therapy" & 109 (23.7) \\
\hline 0 & $375(81.7)$ & Presence of renal calculus** & $37(8.1)$ \\
\hline $1-3$ & $70(15.3)$ & Partially treated UTI & $99(21.6)$ \\
\hline$>3$ & $14(3.1)$ & Renal punch & \\
\hline Past history & & Positive & $400(87.1)$ \\
\hline Diabetes mellitus & $80(17.4)$ & Equivocal & $28(6.1)$ \\
\hline Hypertension & $68(14.8)$ & Abdominal tenderness & $226(49.2)$ \\
\hline Cancer & $11(2.4)$ & Reviewed by gynaecologist & $35(7.6)$ \\
\hline Immunocompromised & $4(0.9)$ & Intravenous fluids & $423(92.2)$ \\
\hline Urinary tract abnormality & $56(12.2)$ & Antibiotics & $420(91.5)$ \\
\hline Temperature ${ }^{\ddagger}\left({ }^{\circ} \mathrm{C}\right)$ & $38.0 \pm 1.1$ & Analgesia & $390(85.0)$ \\
\hline Heart rate ${ }^{\ddagger}$ (beats/min) & $102 \pm 19$ & Disposition & \\
\hline Systolic blood pressure ${ }^{\ddagger}(\mathrm{mmHg})$ & $119 \pm 21$ & Discharged from ED & $35(7.6)$ \\
\hline Diastolic blood pressure ${ }^{\ddagger}(\mathrm{mmHg})$ & $73 \pm 11$ & EDOU & $164(35.7)$ \\
\hline Mean arterial pressure ${ }^{\ddagger}(\mathrm{mmHg})$ & $87 \pm 13$ & General ward & $236(51.4)$ \\
\hline Respiratory rate* (breaths/min) & $18(18-19)$ & $\mathrm{HD} / \mathrm{ICU}$ & $8(1.7)$ \\
\hline Symptom & & Discharged against advice & $16(3.5)$ \\
\hline Presence of flank pain & 439 (95.6) & & \\
\hline
\end{tabular}

*Data presented as median (interquartile range). †Defined as $\geq 2$ infections in six months or $\geq 3$ infections in one year. $\neq$ Data presented as mean \pm standard deviation. §Defined as abnormal vaginal discharge, dyspareunia or abnormal uterine bleeding. १Defined as prior visit to primary care physician and receiving treatment (e.g. antibiotics, analgesia, symptomatic treatment). **Based on known history of renal calculi determined via medical records. ED: emergency department; EDOU: emergency department observation unit; HD/ICU: high dependency/intensive care unit; UTI: urinary tract infection

logistic regression identified the presence of vomiting (OR 4.45, $95 \% \mathrm{Cl}$ 1.45-13.66) as the only remaining significant factor associated with a higher probability of failure of EDOU for acute pyelonephritis.

$84(51.2 \%)$ of the 164 patients admitted to the EDOU had blood cultures done, of which only eight were positive: seven for Escherichia coli and one for Bacillus species (which was deemed to be a contaminant). Among the 459 patients, the $144(31.4 \%)$ patients who were finally classified as having pyelonephritis had a negative urine culture.

Patients who had EDOU success had a higher temperature, heart rate, neutrophil count and urine dipstick nitrite positive rate compared to those discharged directly from the ED, although the association was not clinically significant (Table IV). Among the 51 patients who were discharged directly from the ED, $5(9.8 \%)$ were admitted to the general ward within one week. Out of the 164 patients who were discharged from the EDOU, 2
$(1.2 \%)$ revisited the ED within one week, and one of them was re-admitted to hospital.

\section{DISCUSSION}

In our study, the EDOU acute pyelonephritis protocol was shown to be useful for treatment and observation for evolving symptoms and signs. Over one-third of patients presenting to our ED with pyelonephritis were managed in the EDOU. To the best of our knowledge, there is little data in the literature regarding EDOU admission rates for pyelonephritis in other centres. Schrock and colleagues demonstrated an EDOU admission rate of $24 \%$, but their EDOU was newly created when the data was collected, which possibly accounted for the lower rate. ${ }^{(12)}$

Unresolved fever was the most commonly cited reason for EDOU failure in the present study. Of note, unresolved fever is not listed as an admission criterion in our protocol and some patients were successfully discharged from the ED despite unresolved 
Table II. Laboratory and radiological investigations.

\begin{tabular}{|c|c|c|}
\hline Variable & No. of results & No. (\%) \\
\hline \multicolumn{3}{|l|}{ Laboratory results } \\
\hline White cell count ${ }^{*}\left(\times 10^{9} / \mathrm{L}\right)$ & 453 & $14.07 \pm 5.05$ \\
\hline Neutrophil count* $\left(\times 10^{9} / \mathrm{L}\right)$ & 453 & $11.69 \pm 5.45$ \\
\hline Urea $^{\dagger}(\mathrm{mmol} / \mathrm{L})$ & 452 & $3.7(2.8-4.8)$ \\
\hline Creatinine $^{\dagger}(\mu \mathrm{mol} / \mathrm{L})$ & 452 & $65(55-82)$ \\
\hline Lactate $^{\dagger}(\mathrm{mmol} / \mathrm{L})$ & 120 & $1.3(0.9-2.0)$ \\
\hline Blood cultures & 312 & \\
\hline No growth & & $257(82.4)$ \\
\hline Escherichia coli & & $44(14.1)$ \\
\hline Klebsiella pneumoniae & & $5(1.6)$ \\
\hline Others & & $6(1.9)$ \\
\hline Urine dipstick white blood cell & 405 & \\
\hline 0 & & $35(8.6)$ \\
\hline $1+$ & & $37(9.1)$ \\
\hline $2+$ & & $76(18.8)$ \\
\hline $3+$ & & $257(63.5)$ \\
\hline Urine dipstick nitrite positive & 407 & $168(41.3)$ \\
\hline Urine culture & 434 & \\
\hline No growth & & $144(33.2)$ \\
\hline Escherichia coli & & $233(53.7)$ \\
\hline Klebsiella pneumoniae & & $13(3.0)$ \\
\hline Others & & $45(10.4)$ \\
\hline Not done & & $24(5.5)$ \\
\hline US kidneys $^{\ddagger}$ & 459 & $93(20.3)$ \\
\hline CT KUB & 459 & $6(1.3)$ \\
\hline CT abdomen and pelvis & 459 & $42(9.2)$ \\
\hline
\end{tabular}

Data presented as *mean \pm standard deviation and tmedian (interquartile range). ‡Performed either by radiology or emergency department physicians. $\mathrm{CT}$ : computed tomography; KUB: kidneys, ureters and bladder; US: ultrasonography

fever. The reason for admitting patients with unresolved fever was not always documented in the case records. Based on clinical experience, we postulate that unresolved fever is associated with an overall fatigued and dehydrated state, and such patients tend to have poor oral intake, which indirectly leads to EDOU failure. In the local population, patients have expectations that fever should lyse by the time they are discharged, and patients may not feel confident of managing their illness at home if they are still febrile when leaving the EDOU, leading to increased admissions. However, it is not always possible to predict which group of patients will end up getting admitted, as there are no clear predictors to indicate the pattern of fever.

The results of this study suggest that fewer patients in the EDOU success group tend to have nausea or vomiting. We note that the inability to retain feeds was not the top reason for EDOU failure in this study. The decision for admission is often influenced by a constellation of factors, as illustrated in this study, and we postulate that these factors may not have been adequately documented in the case records. As listed in the discharge criteria (Appendix), patients should be able to retain feeds before discharge, which may explain why fewer patients in the EDOU success group have nausea or vomiting. Hence, for patients with pronounced symptoms of vomiting on presentation to the ED, we strongly recommend routine administration of antiemetics to control symptoms, with consideration of second-line therapy if the symptoms of vomiting remain protracted. These measures may help to maximise EDOU effectiveness.

In our study, only a minority $(11.1 \%)$ of patients were discharged from the ED, in contrast to results from overseas centres. Possible explanations include patient expectations and the perceived risk of failure of outpatient therapy with subsequent reattendance. The patient characteristics of those discharged directly from the ED were largely similar to those with EDOU success. This gives rise to the question of whether these patients could potentially have been discharged directly from the ED. However, it would be difficult to predict which of the patients fall into this category given the relatively high failure rate in the EDOU.

$144(31.4 \%)$ patients who were finally classified as having pyelonephritis had a negative urine culture. The percentage of patients with negative urine cultures in other studies ranged from $3.6 \%$ to $32.3 \%$ depending on the inclusion and exclusion criteria of each study. ${ }^{(7,11)}$ The high percentage of negative urine cultures found could be due to 109 (23.7\%) patients having received outpatient antibiotics before the $\mathrm{ED}$ visit, although the details of the type of antibiotics and duration of treatment were often unspecified. Another reason could be the commencement of empirical antibiotics in the ED after obtaining blood cultures, with a delay in obtaining urine cultures, particularly for patients who appeared ill. A previous study had shown that negative urine cultures can coexist with imaging-proven pyelonephritis. ${ }^{(13)}$

For patients with uncomplicated pyelonephritis, blood cultures are not routinely recommended, as the results do not alter clinical management and seldom vary from urine culture results. ${ }^{(12)}$ From previous studies, the discordance between blood and urine cultures does not exceed 3\%.(14,15) Uncomplicated pyelonephritis is defined as pyelonephritis occurring in young females without structural or functional urinary tract abnormalities and without relevant comorbidities, which fits the general characteristics of the vast majority of patients in this study. ${ }^{(16-18)}$ Over half of the patients admitted to the EDOU had blood cultures done; only eight were positive, of which two had uncomplicated pyelonephritis. The results of the blood cultures did not alter the antibiotic regimen of all eight patients. Given the cost associated with negative blood cultures as well as the risk of false positives, it would be prudent to avoid collecting blood cultures if they are not clinically indicated.

From our centre's data on the antibiotic resistance pattern of Enterobacteriaceae for community-acquired urinary tract infections in 2011, 29.8\% of patients were resistant to ciprofloxacin and 33\% were resistant to cotrimoxazole. Our laboratory did not report on the presence of extended-spectrum beta-lactamase producers but only on carbapenem resistance. As a result of emerging resistance, ciprofloxacin is only reserved for cases of pyelonephritis that require deep tissue penetration. Nitrofurantoin had a low resistance rate of $1 \%$ and hence was 
Table III. Characteristics of the EDOU patients.

\begin{tabular}{|c|c|c|c|c|}
\hline \multirow[t]{2}{*}{ Characteristic } & \multicolumn{3}{|c|}{ No. (\%)/median (IQR) } & \multirow[t]{2}{*}{ p-value } \\
\hline & All EDOU patients $(n=164)$ & Success $(n=100)$ & Failure $(n=64)$ & \\
\hline Age (yr) & $30(25-41)$ & $31(25-42)$ & $30(25-41)$ & 0.71 \\
\hline Female gender & $156(95.1)$ & $93(93.0)$ & $63(98.4)$ & 0.11 \\
\hline Ethnicity & & & & 0.98 \\
\hline Chinese & & $44(44.0)$ & $29(45.3)$ & \\
\hline Malay & & $10(10.0)$ & $5(7.8)$ & \\
\hline Indian & & $25(25.0)$ & $16(25.0)$ & \\
\hline Others & & $21(21.0)$ & $14(21.9)$ & \\
\hline History of pyelonephritis & $15(9.1)$ & $8(8.0)$ & $7(10.9)$ & 0.52 \\
\hline Previous admission for pyelonephritis & $14(8.5)$ & $8(8.0)$ & $6(9.4)$ & 0.76 \\
\hline Episodes of recurrent pyelonephritis & & & & 0.91 \\
\hline 0 & $150(91.5)$ & $92(92.0)$ & $58(90.6)$ & \\
\hline $1-3$ & $14(8.5)$ & $8(8.0)$ & $6(9.4)$ & \\
\hline \multicolumn{5}{|l|}{ Past history } \\
\hline Diabetes mellitus & $10(6.1)$ & $6(6.0)$ & $4(4.0)$ & 0.95 \\
\hline Hypertension & $10(6.1)$ & $8(8.0)$ & $2(3.1)$ & 0.20 \\
\hline Cancer & $1(0.6)$ & $1(1.0)$ & $0(0)$ & 0.42 \\
\hline Immunocompromised & $1(0.6)$ & $1(1.0)$ & $0(0)$ & 0.42 \\
\hline Urinary tract abnormality & $2(1.2)$ & $1(1.0)$ & $1(1.6)$ & 0.75 \\
\hline Previous UTI & $33(20.1)$ & $18(18.0)$ & $15(23.4)$ & 0.40 \\
\hline Recurrent UTI* & $13(7.9)$ & $6(6.0)$ & $7(10.9)$ & 0.38 \\
\hline Temperature $\left({ }^{\circ} \mathrm{C}\right)$ & $38.2(37.5-38.9)$ & $38.2(37.3-38.9)$ & $38.3(37.6-38.8)$ & 0.37 \\
\hline Heart rate (beats/min) & $101(94-114)$ & $100(93-113)$ & $106(95-114)$ & 0.39 \\
\hline Systolic blood pressure $(\mathrm{mmHg})$ & $112(103-124)$ & $114(105-125)$ & $107(100-120)$ & 0.03 \\
\hline Diastolic blood pressure $(\mathrm{mmHg})$ & $73(66-79)$ & $73(66-79)$ & $71(65-77)$ & 0.07 \\
\hline Mean arterial pressure $(\mathbf{m m H g})$ & $84(78-92)$ & $86(78-93)$ & $82(76-90)$ & 0.06 \\
\hline Respiratory rate (breaths/min) & $18(17-19)$ & $18(17-19)$ & $18(17-19)$ & 0.51 \\
\hline \multicolumn{5}{|l|}{ Symptoms } \\
\hline Flank pain & $161(98.2)$ & $99(99.0)$ & $62(96.9)$ & 0.32 \\
\hline Fever & $155(94.5)$ & $91(91.0)$ & $64(100.0)$ & 0.01 \\
\hline Chills & $58(35.4)$ & $32(32.0)$ & $26(40.6)$ & 0.54 \\
\hline Nausea & $71(43.3)$ & $32(32.0)$ & $39(60.9)$ & $<0.001$ \\
\hline Vomiting & $47(28.7)$ & $15(15.0)$ & $32(50.0)$ & $<0.001$ \\
\hline Dysuria & $131(79.9)$ & $82(82.0)$ & $49(76.6)$ & 0.40 \\
\hline Abdominal pain & $78(47.6)$ & $47(47.0)$ & $31(48.4)$ & 0.86 \\
\hline Gynaecological-related complaints $^{\dagger}$ & $7(4.3)$ & $5(5.0)$ & $2(3.1)$ & 0.56 \\
\hline Duration of symptoms (day) & $3(2-5)$ & $3(2-5)$ & $3(2-5)$ & 0.95 \\
\hline Site of flank pain & & & & 0.45 \\
\hline Right & $99(60.4)$ & $57(57.0)$ & $42(65.6)$ & \\
\hline Left & $53(32.3)$ & $36(36.0)$ & $17(26.6)$ & \\
\hline Both & $12(7.3)$ & $7(7.0)$ & $5(7.8)$ & \\
\hline Underwent outpatient therapy ${ }^{\ddagger}$ & $43(26.2)$ & $26(26.0)$ & $17(26.6)$ & 0.94 \\
\hline Presence of renal calculus ${ }^{\S}$ & $2(1.2)$ & $2(2.0)$ & $0(0)$ & 0.26 \\
\hline Partially treated UTI & $38(23.2)$ & $23(23.0)$ & $15(23.4)$ & 0.95 \\
\hline Renal punch & & & & 1.00 \\
\hline Positive & $151(92.1)$ & $91(91.0)$ & $60(93.8)$ & \\
\hline Equivocal & $4(2.4)$ & $3(3.0)$ & $1(1.6)$ & \\
\hline Absent & $9(5.5)$ & $6(6.0)$ & $3(4.7)$ & \\
\hline Abdominal tenderness & $79(48.2)$ & $47(47.0)$ & $32(50.0)$ & 0.71 \\
\hline Reviewed by O\&G & $8(4.9)$ & $5(5.0)$ & $3(4.7)$ & 0.93 \\
\hline
\end{tabular}


Table III. (Contd...)

\begin{tabular}{|c|c|c|c|c|}
\hline \multirow[t]{2}{*}{ Characteristic } & \multicolumn{3}{|c|}{ No. (\%)/median (IQR) } & \multirow[t]{2}{*}{ p-value } \\
\hline & All EDOU patients $(n=164)$ & Success $(n=100)$ & Failure $(n=64)$ & \\
\hline \multicolumn{5}{|l|}{ Laboratory result } \\
\hline White cell count $\left(\times 10^{9} / L\right)$ & $13.76(11.40-17.17)$ & $12.88(10.64-15.66)$ & $15.09(12.19-17.82)$ & 0.007 \\
\hline Neutrophil count $\left(\times 10^{9} / \mathrm{L}\right)$ & $11.27(8.55-14.54)$ & $10.90(8.20-13.3)$ & $12.84(9.90-15.35)$ & 0.007 \\
\hline Neutrophil proportion (\%) & $83.7(76.6-87.4)$ & $82.3(74.8-87.5)$ & $84.2(79.5-87.3)$ & 0.18 \\
\hline Urea (mmol/L) & $3.4(2.7-4.2)$ & $3.2(2.7-4.1)$ & $3.6(2.9-4.7)$ & 0.006 \\
\hline Creatinine $(\mu \mathrm{mol} / \mathrm{L})$ & $62(54-73)$ & $60(54-72)$ & $63(56-74)$ & 0.24 \\
\hline Blood culture & & & & $<0.001$ \\
\hline No growth & $76(46.3)$ & $31(31.0)$ & $45(70.3)$ & \\
\hline Escherichia coli & $7(4.3)$ & $0(0)$ & $7(10.9)$ & \\
\hline Others & $1(0.6)$ & $0(0)$ & $1(1.6)$ & \\
\hline Not done & $80(48.8)$ & $69(69.0)$ & $11(17.2)$ & \\
\hline Urine dipstick white blood cell & $(n=147)$ & $(\mathrm{n}=90)$ & $(n=57)$ & 0.32 \\
\hline 0 & $9(6.1)$ & $7(7.8)$ & $2(3.5)$ & \\
\hline $1+$ & $13(8.8)$ & $6(6.7)$ & $7(12.3)$ & \\
\hline $2+$ & $20(13.6)$ & $10(11.1)$ & $10(17.5)$ & \\
\hline $3+$ & $105(71.4)$ & $67(74.4)$ & $38(66.7)$ & \\
\hline Urine dipstick nitrite positive & $(n=150)$ & $(n=92)$ & $(n=58)$ & 0.41 \\
\hline Urine culture & & & & 0.237 \\
\hline No growth & $47(28.7)$ & $32(32.0)$ & $15(23.4)$ & \\
\hline Escherichia coli & $106(64.6)$ & $61(61.0)$ & $45(70.3)$ & \\
\hline Others & $10(6.1)$ & $6(6.0)$ & $4(6.3)$ & \\
\hline Not done & $1(0.6)$ & $1(1.0)$ & $0(0)$ & \\
\hline US kidneys & $25(15.2)$ & $12(12.0)$ & $13(20.3)$ & 0.15 \\
\hline CT abdomen and pelvis & $7(4.3)$ & $4(4.0)$ & $3(4.7)$ & 0.83 \\
\hline Intravenous fluids & $157(95.7)$ & $95(95.0)$ & $62(96.9)$ & 0.56 \\
\hline Antibiotics & $158(96.3)$ & $96(96.0)$ & $62(96.9)$ & 0.77 \\
\hline Analgesia & $145(88.4)$ & $86(86.0)$ & $59(92.2)$ & 0.23 \\
\hline \multicolumn{5}{|l|}{ EDOU management } \\
\hline Antibiotics & $164(100.0)$ & & & \\
\hline Analgesia & $164(100.0)$ & & & \\
\hline Intravenous fluids & $160(97.6)$ & $98(98.0)$ & $62(96.9)$ & 0.64 \\
\hline US kidneys & $34(20.7)$ & $20(20.0)$ & $14(21.9)$ & 0.84 \\
\hline CT KUB & $6(3.7)$ & $4(4.0)$ & $2(3.1)$ & 1.00 \\
\hline CT abdomen and pelvis & $8(4.9)$ & $2(2.0)$ & $6(9.4)$ & 0.06 \\
\hline Length of stay (day) & $1(1-4)$ & $1(1)$ & $4(3-6)$ & $<0.001$ \\
\hline
\end{tabular}

Value of $\mathrm{n}$ is stated in cases where some data was unavailable. *Defined as $\geq 2$ infections in six months or $\geq 3$ infections in one year. tDefined as abnormal vaginal discharge, dyspareunia, abnormal uterine bleeding. ₹Prior visit to primary care physician and receiving treatment (e.g. antibiotics, analgesia, symptomatic treatment). §Known history of renal calculi determined via medical records. CT: computed tomography; EDOU: emergency department observation unit; IQR: interquartile range; KUB: kidneys, ureters and bladder O\&G: obstetrics and gynaecology department; US: ultrasonography; UTI: urinary tract infection

deemed the antibiotic of choice for simple urinary tract infections, in line with local recommendations. ${ }^{(19}$

This study had several limitations. First, given the setup of our national electronic healthcare records system, we were unable to ascertain if patients included in the study had been re-admitted to hospitals that were not restructured government hospitals, which would have skewed the results towards successful EDOU treatment. However, we postulate that this proportion would be very low, as almost all patients who were admitted to the EDOU received healthcare subsidies and were likely to opt to continue receiving them. Second, this was a single-centre retrospective study and had missing data on certain variables. As a single-centre study, the results may not be reproducible in other healthcare settings or apply to institutions with different patient demographics and prevalence of pyelonephritis. In addition, variables that had not been documented were regarded as being absent, and it is likely that the missing data led to underreporting of potential symptoms related to pyelonephritis. 
Table IV. Comparison of patients discharged from the ED and those in the EDOU success group.

\begin{tabular}{|c|c|c|c|}
\hline \multirow[t]{2}{*}{ Characteristic } & \multicolumn{2}{|c|}{ No. (\%)/median (IQR) } & \multirow[t]{2}{*}{ p-value } \\
\hline & Discharged from ED $(n=35)$ & EDOU success $(n=100)$ & \\
\hline Age (yr) & $33(27-44)$ & $31(25-41.5)$ & 0.25 \\
\hline Female gender & $31(88.6)$ & $93(93.0)$ & 0.41 \\
\hline Ethnicity & & & 0.65 \\
\hline Chinese & $13(37.1)$ & $44(44.0)$ & \\
\hline Malay & $5(14.3)$ & $10(10.0)$ & \\
\hline Indian & $7(20.0)$ & $25(25.0)$ & \\
\hline Others & $10(28.6)$ & $21(21.0)$ & \\
\hline History of pyelonephritis & $9(25.7)$ & $8(8.0)$ & 0.01 \\
\hline Previous admission for pyelonephritis & $8(22.9)$ & $8(8.0)$ & 0.03 \\
\hline Episodes of recurrent pyelonephritis & & & 0.02 \\
\hline 0 & $26(74.3)$ & $92(92.0)$ & \\
\hline $1-3$ & $6(17.1)$ & $8(8.0)$ & \\
\hline$>3$ & $3(8.6)$ & $0(0)$ & \\
\hline \multicolumn{4}{|l|}{ Past history } \\
\hline Diabetes mellitus & $4(11.4)$ & $6(6.0)$ & 0.28 \\
\hline Hypertension & $4(11.4)$ & $8(8.0)$ & 0.51 \\
\hline Cancer & $0(0)$ & $1(1.0)$ & 1.00 \\
\hline Immunocompromised & $0(0)$ & $1(1.0)$ & 1.00 \\
\hline Urinary tract abnormality & $1(2.9)$ & $1(1.0)$ & 0.45 \\
\hline Previous UTI & $12(34.3)$ & $18(18.0)$ & 0.06 \\
\hline Recurrent UTI* & $7(20.0)$ & $6(6.0)$ & 0.03 \\
\hline Temperature $\left({ }^{\circ} \mathrm{C}\right)$ & $37.6(36.7-38.1)$ & $38.2(37.3-38.9)$ & 0.01 \\
\hline Heart rate (beats/min) & $93(84-108)$ & $100(93-113)$ & 0.01 \\
\hline Systolic blood pressure (mmHg) & $114(107-136)$ & $114(105-125)$ & 0.24 \\
\hline Diastolic blood pressure $(\mathrm{mmHg})$ & $73(68-84)$ & $73(66-79)$ & 0.56 \\
\hline Mean arterial pressure $(\mathrm{mmHg})$ & $86.5(81.5-98.0)$ & $85.8(78.2-93.4)$ & 0.32 \\
\hline Respiratory rate (breaths/min) & $18(18-19)$ & $18(17-19)$ & 0.36 \\
\hline \multicolumn{4}{|l|}{ Symptom } \\
\hline Flank pain & $35(100.0)$ & $99(99.0)$ & 1.00 \\
\hline Fever & $33(94.3)$ & $91(91.0)$ & 0.73 \\
\hline Chills & $7(20.0)$ & $32(32.0)$ & 0.37 \\
\hline Nausea & $10(28.6)$ & $32(32.0)$ & 0.83 \\
\hline Vomiting & $6(17.1)$ & $15(15.0)$ & 0.79 \\
\hline Dysuria & $29(82.9)$ & $82(82.0)$ & 1.00 \\
\hline Abdominal pain & $13(37.1)$ & $47(47.0)$ & 0.33 \\
\hline Gynaecological-related complaints $^{\dagger}$ & $0(0)$ & $5(5.0)$ & 0.33 \\
\hline Duration of symptoms (day) & $3(2-4)$ & $3(2-5)$ & \\
\hline Site of flank pain & $(n=34)$ & & 0.47 \\
\hline Right & $16(47.1)$ & $57(57.0)$ & \\
\hline Left & $14(41.2)$ & $36(36.0)$ & \\
\hline Both & $4(11.8)$ & $7(7.0)$ & \\
\hline Underwent outpatient therapy ${ }^{\ddagger}$ & $7(20.0)$ & $26(26.0)$ & 0.65 \\
\hline Presence of renal calculus ${ }^{\S}$ & $1(2.9)$ & $2(2.0)$ & 1.00 \\
\hline Partially treated UTI & $5(14.3)$ & $23(23.0)$ & 0.34 \\
\hline Renal punch & & & 0.01 \\
\hline Positive & $28(80.0)$ & $91(91.0)$ & \\
\hline Equivocal & $6(17.1)$ & $3(3.0)$ & \\
\hline Absent & $1(2.9)$ & $6(6.0)$ & \\
\hline
\end{tabular}


Table IV. (Contd...)

\begin{tabular}{|c|c|c|c|}
\hline \multirow[t]{2}{*}{ Characteristic } & \multicolumn{2}{|c|}{ No. (\%)/median (IQR) } & \multirow[t]{2}{*}{ p-value } \\
\hline & Discharged from ED $(n=35)$ & EDOU success $(n=100)$ & \\
\hline Abdominal tenderness & $13(37.1)$ & $47(47.0)$ & 0.33 \\
\hline Reviewed by O\&G & $1(2.9)$ & $5(5.0)$ & 1.00 \\
\hline \multicolumn{4}{|l|}{ Laboratory result } \\
\hline White cell count $\left(\times 10^{9} / L\right)$ & $12.27(9.60-13.73)$ & $12.88(10.64-15.66)$ & 0.07 \\
\hline Neutrophil count $\left(\times 10^{9} / \mathrm{L}\right)$ & $9.91(6.94-11.24)$ & $10.90(8.20-13.3)$ & 0.03 \\
\hline Neutrophil proportion (\%) & $78.8(72.5-88.2)$ & $82.3(74.8-87.5)$ & 0.94 \\
\hline Urea $(\mathrm{mmol} / \mathrm{L})$ & $3.2(2.5-3.8)$ & $3.2(2.7-4.1)$ & 0.20 \\
\hline Creatinine ( $\mu \mathrm{mol} / \mathrm{L})$ & $59(53-64)$ & $60(54-72)$ & 0.04 \\
\hline Blood culture & & & 0.83 \\
\hline No growth & $9(25.7)$ & $31(31.0)$ & \\
\hline Escherichia coli & $0(0)$ & $0(0)$ & \\
\hline Others & $0(0)$ & $0(0)$ & \\
\hline Not done & $26(74.3)$ & $69(69.0)$ & \\
\hline Urine dipstick white blood cell & $(n=31)$ & $(n=90)$ & 0.18 \\
\hline 0 & $4(12.9)$ & $7(7.8)$ & \\
\hline $1+$ & $4(12.9)$ & $6(6.7)$ & \\
\hline $2+$ & $4(12.9)$ & $10(11.1)$ & \\
\hline $3+$ & $19(61.3)$ & $67(74.4)$ & \\
\hline Urine dipstick nitrite positive & $(n=32) 7(21.9)$ & $(n=92) 41(44.6)$ & 0.01 \\
\hline Urine culture & & & $<0.001$ \\
\hline No growth & $28(80.0)$ & $32(32.0)$ & \\
\hline Escherichia coli & $1(2.9)$ & $61(61.0)$ & \\
\hline Others & $6(17.1)$ & $6(6.0)$ & \\
\hline Not done & $0(0)$ & $1(1.0)$ & \\
\hline US kidneys & $0(0)$ & $12(12.0)$ & 0.01 \\
\hline CT abdomen and pelvis & $3(8.6)$ & $4(4.0)$ & 0.05 \\
\hline Intravenous fluids & $31(88.6)$ & $95(95.0)$ & 1.00 \\
\hline Antibiotics & $33(94.3)$ & $96(96.0)$ & 0.16 \\
\hline Analgesia & $27(77.1)$ & $86(86.0)$ & 0.46 \\
\hline
\end{tabular}

Value of $\mathrm{n}$ is stated in cases where some data was unavailable. *Defined as $\geq 2$ infections in six months or $\geq 3$ infections in one year. tDefined as abnormal vaginal discharge, dyspareunia, abnormal uterine bleeding. ‡Prior visit to primary care physician and receiving treatment (e.g. antibiotics, analgesia, symptomatic treatment). §Known history of renal calculi determined via medical records. CT: computed tomography; ED: emergency department; EDOU: emergency department observation unit; IQR: interquartile range; O\&G: obstetrics and gynaecology; UTI: urinary tract infection; US: ultrasonography

Lastly, we relied upon clinical details, which can be ambiguous and challenging, to define the diagnosis. ${ }^{(13,20)}$ Furthermore, the reviewers were not blinded to the study purpose, a possible source of selection bias. Our inclusion criteria may have been overly strict, as we wanted a high degree of certainty in the diagnosis. While the criteria were in line with the existing definition of acute pyelonephritis, patients may also present atypically, especially those who are pretreated with antibiotics in the outpatient setting, or geriatric patients. ${ }^{(21)}$ This limitation may lead to misclassification bias, such that patients with acute pyelonephritis may not have been recognised. However, the misclassification would be non-differential in nature and had a similar chance of being in the EDOU success or failure groups.

In conclusion, the EDOU has been shown to be useful for both treatment of and observation for evolving signs of acute pyelonephritis in order to refine the eventual diagnosis. In our study, successful management in the EDOU was achieved in $100(61.0 \%)$ patients. Urine cultures were sufficient for the identification of the culprit micro-organism. Patients who present with prominent symptoms of vomiting should have routine administration of antiemetics, while consideration for second-line antiemetics is recommended for those with persistent symptoms. Controlling symptoms of vomiting would promote better oral intake and may increase EDOU discharge rates.

\section{SUPPLEMENTARY MATERIAL}

The Appendix is available online at https://doi.org/10.11622/ smedj.2020020.

\section{REFERENCES}

1. Fairley KF, Carson NE, Gutch RC, et al. Site of infection in acute urinary-tract infection in general practice. Lancet 1971; 2:615-8.

2. Stamm WE, Norrby SR. Urinary tract infections: disease panorama and challenges. J Infect Dis 2001; 183 Suppl 1:S1-4. 
3. Brown P, Ki M, Foxman B. Acute pyelonephritis among adults: cost of illness and considerations for the economic evaluation of therapy. Pharmacoeconomics 2005; 23:1123-42.

4. Czaja CA, Scholes D, Hooton TM, Stamm WW. Population-based epidemiologic analysis of acute pyelonephritis. Clin Infect Dis 2007; 45:273-80.

5. van Nieuwkoop C, van't Wout JW, Spelt IC, et al. Prospective cohort study of acute pyelonephritis in adults: safety of triage towards home based oral antimicrobial treatment. J Infect 2010; 60:114-21.

6. Safrin S, Siegel D, Black D. Pyelonephritis in adult women: inpatient versus outpatient therapy. Am J Med 1988; 85:793-8.

7. Pinson AG, Philbrick JT, Lindbeck GH, Schorling JB. ED management of acute pyelonephritis in women: a cohort study. Am J Emerg Med 1994; 12:271-8.

8. Efstathiou SP, Pefanis AV, Tsioulos DI, et al. Acute pyelonephritis in adults: prediction of mortality and failure of treatment. Arch Intern Med 2003; 163:1206-12.

9. Israel RS, Lowenstein SR, Marx JA, et al. Management of acute pyelonephritis in an emergency department observation unit. Ann Emerg Med 1991; 20:253-7.

10. Ward G, Jorden RC, Severance HW. Treatment of pyelonephritis in an observation unit. Ann Emerg Med 1991; 20:258-61.

11. Buonaiuto V, Marquez I, De Toro I, et al. Clinical and epidemiological features and prognosis of complicated pyelonephritis: a prospective observational single hospital-based study. BMC Infect Dis 2014; 14:639.

12. Schrock JW, Reznikova S, Weller S. The effect of an observation unit on the rate of ED admission and discharge for pyelonephritis. Am J Emerg Med 2010; 28:682-8.

13. Piccoli GB, Consiglio V, Deagostini MC, et al. The clinical and imaging presentation of acute "non complicated" pyelonephritis: a new profile for an ancient disease. BMC Nephrol 2011; 12:68

14. McMurray BR, Wrenn KD, Wright SW. Usefulness of blood cultures in pyelonephritis. Am J Emerg Med 1997; 15:137-40.

15. Chen $Y$, Nitzan $O$, Saliba W, et al. Are blood cultures necessary in the management of women with complicated pyelonephritis? J Infect 2006; 53:235-40.

16. Colgan R, Williams M, Johnson JR. Diagnosis and treatment of acute pyelonephritis in women. Am Fam Physician 2011; 84:519-26.

17. Nicolle LE. Uncomplicated urinary tract infection in adults including uncomplicated pyelonephritis. Urol Clin North Am 2008; 35:1-12, v.

18. Hsu CY, Fang HC, Chou KJ, et al. The clinical impact of bacteremia in complicated acute pyelonephritis. Am J Med Sci 2006; 332:175-80.

19. Tan CW, Chlebicki MP. Urinary tract infections in adults. Singapore Med J 2016; 57:485-90.

20. Talner LB, Davidson AJ, Lebowitz RL, Dalla Palma L, Goldman SM. Acute pyelonephritis: can we agree on terminology? Radiology 1994; 192:297-305.

21. Liang SY, Mackowiak PA. Infections in the elderly. Clin Geriatr Med 2007; 23:441-56 


\section{APPENDIX}

Extended diagnostic observation unit pre-admission criteria for acute pyelonephritis/urinary tract infection

\section{Inclusion criteria}

1. Haemodynamically stable

2. Lactate $<4.0 \mathrm{mmol} / \mathrm{L}$ or falling trend

3. Age $<70$ years

\section{Exclusion criteria}

1. Septic patients/unstable vital signs or lactate $>4.0 \mathrm{mmol} / \mathrm{L}$ or rising trend

2. Ballotable enlarged kidney/pyuria/pyonephrosis/perinephric abscess/obstructive case, e.g. calculi

3. Acute renal failure: serum creatinine $>200 \mu \mathrm{mol} / \mathrm{L}$

4. Renal transplant/immunocompromised/single kidney/known structure defect, e.g. polycystic kidney disease, vesicoureteral reflux, duplex ureter, stricture, neurogenic bladder

5. Multiple comorbidities or multiple acute medical illnesses, poorly controlled diabetes mellitus, chronic obstructive pulmonary disease, malignancy

6. Pregnant

7. Social issues

\section{Emergency department interventions}

1. Full blood count, renal panel including bicarbonate level, lactate

2. Urine analysis, urine culture and sensitivity, blood culture and sensitivity

3. Intravenous normal saline $2-3 \mathrm{~L}$ over $1-2$ hours

4. Intravenous ceftriaxone $2 \mathrm{~g}$ stat (intravenous ciprofloxacin if there is a known allergy to penicillin). Consider one dose of gentamicin $(5 \mathrm{mg} / \mathrm{kg})$ if previously treated and still symptomatic

5. Oral paracetamol $1 \mathrm{~g}$ stat

6. Intravenous metoclopramide $10 \mathrm{mg}$ if vomiting

\section{Emergency department observation unit interventions}

1. Vital signs four-hourly unless specified

2. Intravenous Ringer's lactate solution $500 \mathrm{~mL}$ four-hourly

3. Replace potassium

4. Intravenous ceftriaxone $1 \mathrm{~g}$ twice a day (BD)

Consider one dose of gentamicin $(5 \mathrm{mg} / \mathrm{kg}$ ) if previously treated and still symptomatic

5. Oral paracetamol $1 \mathrm{~g}$ six-hourly

6. Intravenous metoclopramide $10 \mathrm{mg}$ eight-hourly if vomiting

7. Renal ultrasonography if indicated clinically (ballotable/enlarged kidney, pyuria, increasing loin pain)

8. Repeat renal panel and lactate if indicated

9. Trace urine and blood culture and sensitivity

\section{Discharge criteria}

1. Vital signs stable

2. Able to retain feeds (patient with low-grade fever may still be discharged if eating well and decreasing loin pain)

3. Oral ciprofloxacin $500 \mathrm{mg} \mathrm{BD}$ for one week (for pyelonephritis). Avoid use of ciprofloxacin in uncomplicated lower urinary tract infections

4. Oral nitrofurantoin $100 \mathrm{mg} \mathrm{BD}$ for five days or trimethoprim/sulfamethoxazole two tabs BD for three days or cefuroxime/cephalexin/co-amoxiclav for 3-7 days for lower urinary tract infections (tailor according to culture sensitivity if ready)

5. Emergency department observation unit staff nurse to trace blood or urine cultures and sensitivities the next day if results are not ready on discharge

6. Appointment at primary healthcare centre in five days for a repeat renal panel and urine culture

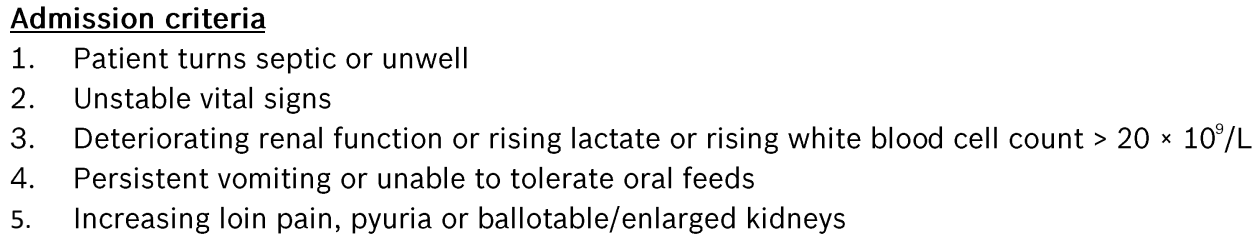

